\title{
Hybrid fiber reinforcement and crack formation in Cementitious Composite Materials
}

\author{
Eduardo B. Pereira
}

ISISE, University of Minho, Guimaraes, Portugal

\section{Gregor Fischer}

Technical University of Denmark, Lyngby, Denmark

\author{
Joaquim A. O. Barros
}

ISISE, University of Minho, Guimaraes, Portugal

\begin{abstract}
The use of different types of fibers simultaneously for reinforcing cementitious matrices is motivated by the concept of a multi-scale nature of the crack propagation process. Fibers with different geometrical and mechanical properties are used to bridge cracks of different sizes from the micro- to the macroscale. In this study, the performance of different fiber reinforced cementitious composites is assessed in terms of their tensile stress-crack opening behavior. The results obtained from this investigation allow a direct quantitative comparison of the behavior obtained from the different fiber reinforcement systems. The research described in this paper shows that the multi-scale conception of cracking and the use of hybrid fiber reinforcements do not necessarily result in an improved tensile behavior of the composite. Particular material design requirements may nevertheless justify the use of hybrid fiber reinforcements.
\end{abstract}

\section{Introduction}

Strain-hardening in tension is a relevant material property for structural applications of cementitious composites. Commonly known as Strain hardening cement composites (SHCC), these materials show benefits in terms of durability and preservation of functional properties of the structural elements. Examples of SHCC 
exist which have been designed and proven to gather these requisites in different perspectives (Brandt 2008). Engineered Cementitious Composites (ECC), a class of cement based materials typically reinforced with Polyvinyl Alcohol (PVA) fibers, is one of the examples of SHCC showing relatively high ductility (between $3 \%$ and $7 \%$ of strain) and average tensile strength of $5 \mathrm{MPa}$ (Fischer and Li 2007, Li 2003).

The recent technological development of a wide variety of fibers has created new opportunities for the development of SHCC. Envisaging an optimal utilization of the materials, either in an economical or environmental perspective, hybridization of the fiber reinforcement has been studied in recent past. The use of fibers of different natures, with distinct geometrical and material properties, has been reported to improve the material features of SHCC (Ahmed et al. 2007, Banthia and Gupta 2004, Lawler et al.2003, Rossi 1997).

One of the main advantages usually assigned to the hybridization of fiber reinforcement is the possibility to restrain cracking at different scales (Banthia and Gupta 2004). Concerning the micromechanics of the composite, the commonly recognized multi-scale nature of concrete should be reflected in the cracking processes. In a simplistic perspective, one can assume that the micro-cracks generated during cracking processes are bridged by smaller fibers and the macro-cracks bridged by the larger fibers (Betterman et al. 1995). A crack necessarily undergoes different stages, from onset to the fully separated state, and a visible crack may be seen as the result of the coalescence of previous randomly oriented and diffusely distributed micro-cracks. It seems, though, that the unambiguous evidence to this mechanism is not easy to unveil. The relation between this hierachized conception of the cracking process in a composite, the different material scales and the mechanisms of crack restraining by the fiber reinforcement and stiffening mechanisms in tension are not clearly established.

The assessment of the tensile stress - crack opening law may help to quantify the contribution of each fiber reinforcing system to the overall tensile composite behavior. The potential synergistic effect resulting from fiber hybridization may also be directly and explicitly quantified. For this purpose, the single crack tension test (SCTT) setup may be used to allow the direct assessment of the tensile stress - crack opening law. As shown in previous work, this procedure focuses on the assessment of the tensile stress-crack opening law of SHCC (Fischer et al. 2007, Pereira et al. 2010). This is done in a direct fashion with the main advantage of allowing the direct characterization of the tensile constitutive behavior of the FRCC without the need of knowing the shape of the tensile stress-crack opening law in advance, as opposed to indirect methods where the constitutive tensile stress-crack opening law is derived by inverse analysis (Chanvillard 1999, Lofgren et al. 2008, Zhang et al. 2010). This feature may become especially relevant for composites with hybrid reinforcement, where fibers are activated at different stages of the cracking sequence which may result in more complex shapes of the tensile stress - crack opening law. This procedure may contribute to a deeper and more accurate characterization of the tensile behavior of hybrid FRCC with evi- 
dent benefits for the structural design with SHCC. Additionally, most of the numerical models for structural design rely on the fracture mode I constitutive law for the material modeling (Kabele 2007, Stang et al. 1995). Furthermore, the material design process also benefits from the direct assessment of the tensile stresscrack opening law. The procedure for the optimization of the different composite components becomes more efficient with the direct quantification of differences between distinct composite systems.

The direct assessment of the tensile stress - crack opening law is expected to allow the direct quantification of the contribution of each fiber reinforcing system to the global tensile behavior of the composite. The main objective is to understand the contribution that the Single Crack Tension Test (SCTT) setup can give towards a better understanding of fracture micro-mechanisms and the tensile performance of hybrid fiber reinforced cement composites.

\section{Experimental program}

In the present work, the tensile stress - crack opening law of different hybrid reinforced cement composites was assessed, focusing mainly on the usefulness of the SCTT test-setup to support the material design of hybrid fiber reinforced cement composites.

Fibers of three different natures were used: PVA (polyvinyl alcohol), PAN (polyacrylnitrile) and PP (polypropylene). The main geometrical and mechanical properties of these fibers are presented in Table 1 . The proportions of each fiber reinforcement type in terms of volume percentage used in all the six composites (C1 to C6) are also represented in Table 1.

Table 1. Main physical properties of fibers and composition of the six formulated composites.

\begin{tabular}{|c|c|c|c|c|c|c|c|c|c|}
\hline \multirow{2}{*}{ Fiber } & \multirow{2}{*}{$\begin{array}{c}\begin{array}{c}\text { Tensile } \\
\text { strength }\end{array} \\
\mathrm{MPa}\end{array}$} & \multirow{2}{*}{$\begin{array}{c}\text { Length } \\
\mathrm{mm}\end{array}$} & \multirow{2}{*}{$\frac{\text { Diam }}{\mu \mathrm{m}}$} & \multicolumn{6}{|c|}{ Fiber reinforcement (\%vol) } \\
\hline & & & & $\mathrm{C} 1$ & $\mathrm{C} 2$ & C3 & $\mathrm{C} 4$ & C5 & C6 \\
\hline PVA 15 & 1600 & 8 & 40.0 & 1 & 2 & 0 & 0 & 1 & 1 \\
\hline PP & 900 & 12 & 40.0 & 0 & 0 & 2.5 & 0 & 1.25 & 0 \\
\hline PAN 1.5 & 826 & 6 & 12.7 & 0 & 0 & 0 & 2 & 0 & 1 \\
\hline
\end{tabular}

For each composite, six specimens were tested in direct tension using the SCTT setup (see Pereira et al. 2010), as represented in Fig. 1 and Fig. 2. 


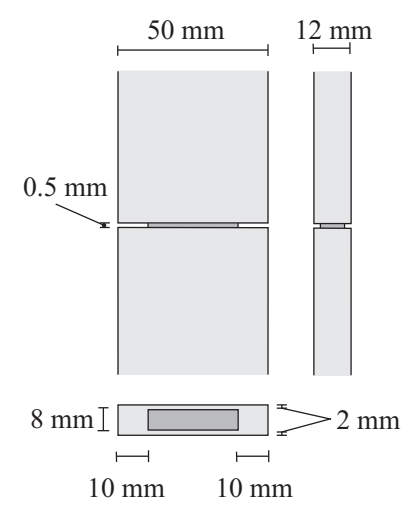

Fig. 1. Geometry used for the assessment of the tensile stress - crack opening law.

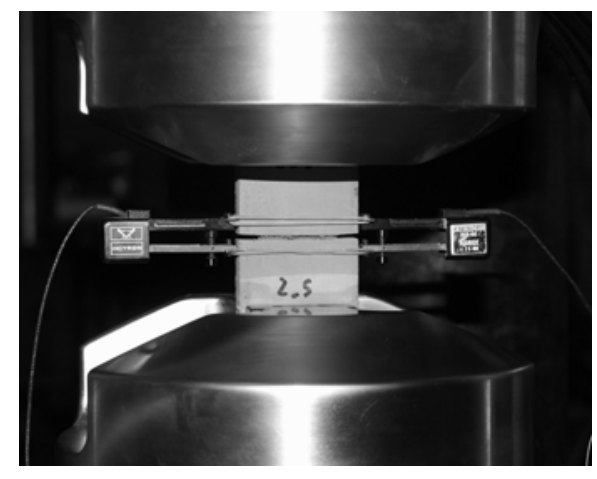

Fig. 2. Tensile test setup, including supports and clip gages.

\section{Results and Discussion}

During the mixing stage, it became evident that the PVA fibers were easier to mix compared to the PAN and PP fibers. As a consequence of the different surface properties of PP fibers, their mixing and dispersion was more difficult. Among all, the PAN fibers were the most difficult to disperse. Likewise, the fibers in the hybrid mixes showed to be easier to mix compared to the single-type fiber reinforced compositions containing PP or PAN fibers.

The test results obtained in terms of tensile stress versus the crack mouth opening displacement (CMOD) for all the composites are presented in Fig. 3 to Fig. 6. The two composites reinforced with PVA fibers (1\% and $2 \%$ ) can be considered as a performance reference given the extensive experience available with PVA ECC (Li 2003). For clarity, only three of the six test results for each composite are presented. The extreme curves were selected for all the composites. The nominal value of the tensile stress was determined by computing the ratio between the registered tensile load during testing and the notched cross section of each specimen.

Relevant information can be extracted from several stages of crack initiation and further opening. In a first overall observation (Fig. 4 and Fig. 6) it becomes apparent that the composite behavior resulting from the mixture of two very distinct types of fibers corresponds approximately to the superposition of the original single-type fiber reinforced composites. At an approximately similar reinforcing percentage, the hybrid composite performs figuratively speaking in between the corresponding original single-type fiber reinforced composites. The different types of fibers, either due to distinct geometrical/mechanical properties or due to different interaction with the surrounding matrix (bonding), showed to be activated distinctively and at different stages of the tensile tests. In Fig. 3 and Fig. 5, the results 
obtained for all the composites for the preliminary stages of cracking are shown. In Fig. 4 and Fig. 6, the overall tensile behavior observed is compared for each group of fibers (PVA/PAN and PVA/PP respectively).

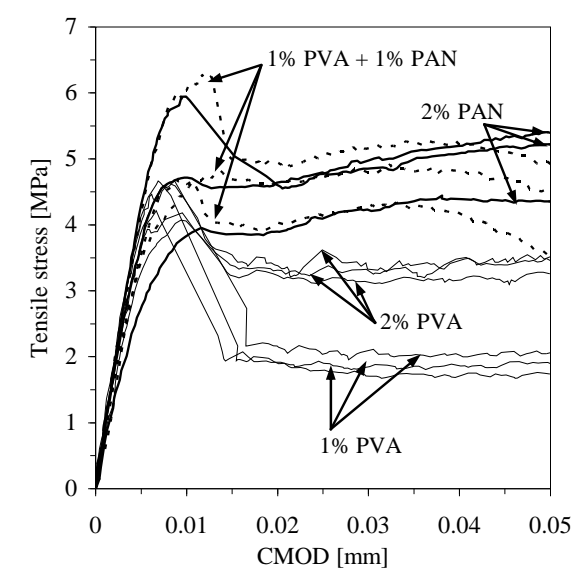

Fig. 3. Preliminary stages of cracking for the composites reinforced with PVA and PAN.

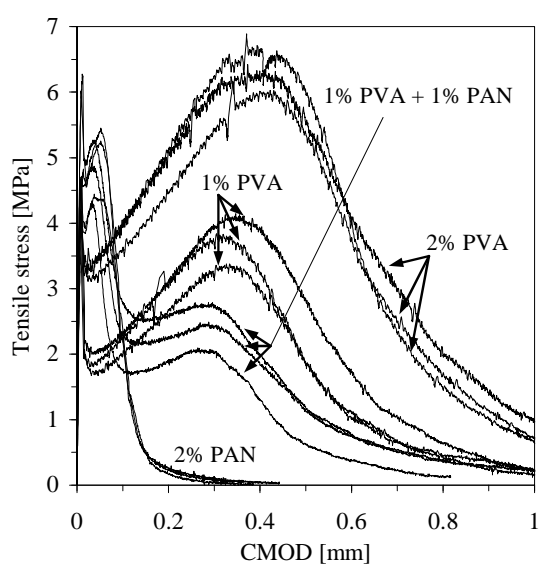

Fig. 4. Overall tensile behavior for the composites reinforced with PVA and PAN.

When using PAN fibers, the fiber reinforcement in the composite is activated at relatively small crack openings. In Fig. 3, one can observe that the composite reinforced with $2 \%$ PAN fibers showed higher first cracking stresses, reaching peak stresses of up to $6 \mathrm{MPa}$ at this stage. The small fiber diameter and the improved bonding with the matrix resulted in a pronounced crack restraining for CMOD smaller than $10 \mu \mathrm{m}$. The subsequent gradual stress decay and the following strain hardening branch developing up to an approximate CMOD of $50 \mu \mathrm{m}$ are a consequence of the gradual mobilization of the PAN fibers present in the composite. After the peak bridging stress is reached, the subsequent stress decay is rapid, as shown in Fig. 4. When half of the volume fraction of the PAN fiber reinforcement is replaced by PVA fibers, some of the crack restraining effect at the early cracking stage is lost. However, the PVA + PAN composite develops a second strain hardening branch for higher CMOD values, replicating closely the strain hardening branch observed in the PVA fiber reinforced composite. It seems that the hybrid fiber composite is able to combine the features of each of the single-type fiber composites. However it always underperforms the single-type fiber composites at the cracking stages where the latter individually revealed to be more effective. For the PAN + PVA composite, this effect was less significant for smaller CMOD (up to $50 \mu \mathrm{m}$ ). For higher CMOD, the PAN + PVA composite underperformed even the composite containing 1\% of PVA. The loss of performance of the fresh mixes due to the addition of PAN fibers may have contributed for these results. 


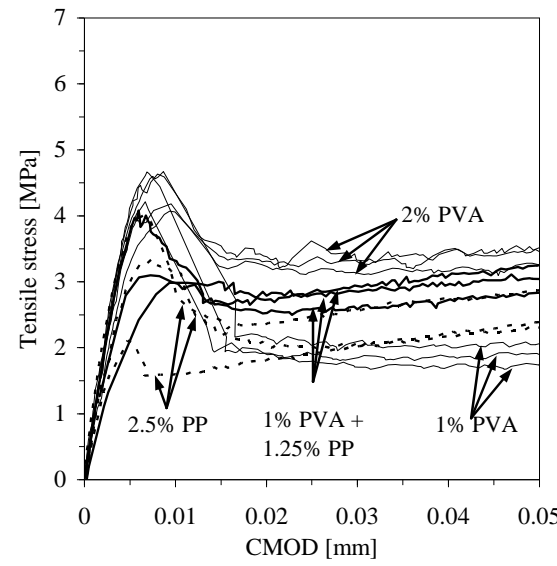

Fig. 5. Preliminary stages of cracking for the composites reinforced with PVA and PP.

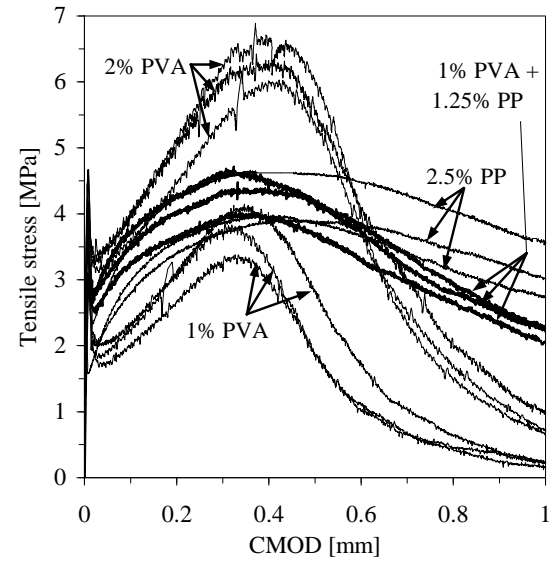

Fig. 6. Overall tensile behavior observed for the composites reinforced with PVA and PP.

On the contrary, the use of the PP fibers resulted in a delayed mobilization of the fiber reinforcement. As shown in Fig. 6, the tensile stress - crack opening curve of the composite reinforced with $2.5 \%$ PP fibers showed a prolonged tail and a high remaining residual tensile stress for a CMOD of $1 \mathrm{~mm}$. Due to the reduced chemical bonding of the fibers with the surrounding matrix, the prevailing pull-out mechanism of PP fibers manifests itself both in the early stages of cracking (Fig. 5) and in the overall behavior (Fig. 6). During the early stage of cracking, the contribution of the PP fibers to the first cracking strength was slightly smaller than the one observed for the PVA reinforced composites. The differences in the fiber properties become more evident beyond the first cracking stage with a more pronounced post-peak load decay observed for the composites reinforced with PP fibers. This may be an indicator of the sudden debonding of the PP fiber at this stage, which is more pronounced than the one taking place in the PVA fibers. The subsequent stress recovery was also steeper for the PP reinforced composites. The premature debonding of the PP fibers was again revealed by the rapid loss of the initial bridging stiffness in the hardening stage. The hybrid composite behavior was found to be a combination of the tensile features observed in the respective single-type fiber composites. As in the case of PAN + PVA, the hybrid composite of PP and PVA underperformed the single-type fiber reinforced composites at the cracking stages where the latter individually revealed to be more effective.

To allow a clearer interpretation of the results above discussed, in Fig. 7 the average of the experimental results obtained for each composite are shown. This was done by averaging the tensile stress obtained for all the six specimens at each CMOD value. An important indicator of the tensile performance of the tested composites is the fracture energy $\left(\mathrm{G}_{\mathrm{f}}\right)$, approximately assessed by computing the area limited under the experimental tensile stress - crack opening curves. These values were computed for the average curves of each composite (see Fig. 8). In 
general, the obtained values show a dispersion that is similar among all the composites, indicating that the parameters adopted for the SCTT setup are adequate and lead to consistent results. The hybrid formulations resulted in tensile performances which are positioned between the ones obtained for the single-type fiber reinforced composites, suggesting that the principle of superposition of the tensile stress - crack opening curves may be used as an underlying mechanism in hybrid FRCC. Considering the potential synergistic effect of having multiple-fiber reinforcements, the hybrid FRCC were expected to outperform the superposition of the respective single-type fiber reinforced composites, which was not observed in this study. In the special case of the hybrid composite reinforced with PVA and PAN fibers, the hybrid even underperformed the 1\% PVA reinforced composite for average CMOD between 0.1 and $0.5 \mathrm{~mm}$. One explanation for this may be the unfavorable effect that PAN fibers have on the fresh properties of the composite.

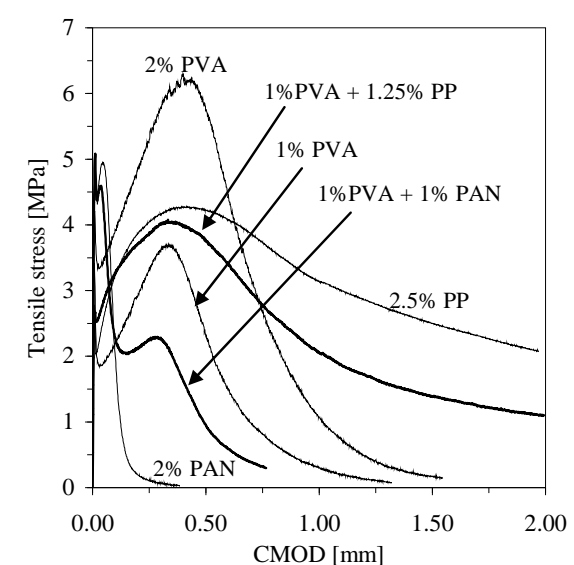

Fig. 7. Overall tensile behavior (average curves).

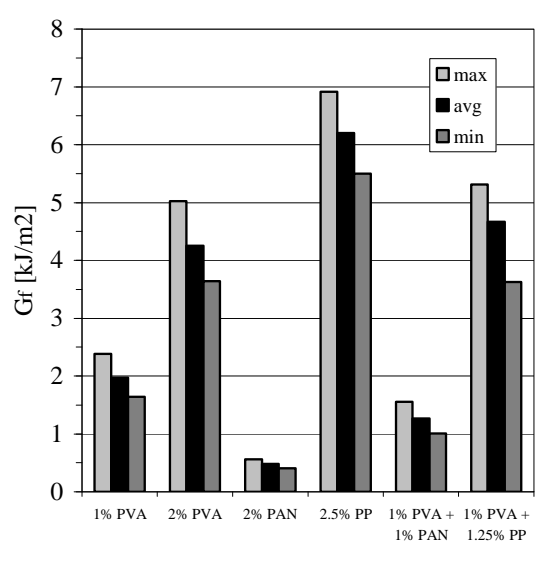

Fig. 8. Fracture energy for the minimum, average and maximum experimental curves.

\section{Conclusions}

In the present work, the single crack tension test (SCTT) setup allowed the unique identification of the mechanical contribution of each fiber reinforcement type on the overall tensile behavior of the hybrid composites. The consistent experimental results support its use for the standard mechanical characterization and design of hybrid FRCC.

The quantitative estimation of the contribution of different fiber types to the overall behavior of hybrid FRCC allowed a better understanding of the principle of superposition of the tensile stress - crack opening curves and the synergistic effect of multi-scale fiber reinforcement for the hybrid tensile micro-mechanisms. The results obtained in this study suggest that the effect of these principles may be 
influenced by other factors. In particular, the fresh properties showed to have strong influence on the overall tensile behavior of the hybrid composites.

\section{Acknowledgments}

The first author thanks the Portuguese National Science Foundation for the financial support, through grant SFRH / BD / 36515 / 2007, funded by POPH QREN, the Social European Fund and the MCTES, and DTU-Byg for their support of the work as part of this project.

\section{References}

Ahmed SFU, Maalej M, Paramasivam P (2007) Flexural responses of hybrid steel-polyethylene fiber reinforced cement composites containing high volume fly ash. Con Build Mat 21:10881097.

Betterman LR, Ouyang C, Shah SP (1995) Fiber-matrix interaction in microfiber reinforced matrix. Adv Cem Bas Mat 2:52-61.

Banthia N, Gupta R (2004) Hybrid fiber reinforced concrete (HyFRC): fiber synergy in high strength matrices. Mat Struct 37:707-716.

Brandt AM (2008) Fibre reinforced cement-based (FRC) composites after over 40 years of development in building and civil engineering. Comp Struct 86: 3-9.

Chanvillard G (1999) Caractérisation des performances d'un béton renforcé de fibres à partir d'un essai de flexion. Partie 2 : Identification d'une loi de comportement intrinsèque en traction. Mat Struct $32: 601-605$.

Fischer G, Li VC (2007) Effect of fiber reinforcement on the response of structural members. Eng Frac Mech 74: 258-272.

Fischer G, Stang H, Dick-Nielsen L (2007) Initiation and development of cracking in ECC materials: Experimental observations and modeling”. In Carpinteri GFA, Gambarova P (eds) High Performance Concrete, Brick- Masonry and Environmental Aspects. Proc int symp, Vol. 3, Ia-FraMCos. Taylor \& Francis, pp. 1517-1522.

Kabele P (2007) Multiscale framework for modeling of fracture in high performance fiber reinforced cementitious composites. Eng Frac Mech 74: 194-209.

Lawler JS, Wilhelm T, Zampini D, Shah SP (2003) Fracture processes of hybrid fiber-reinforced mortar. Mat Struct 36:197-208.

Li VC (2003) On Engineered Cementitious Composites (ECC) - A Review of the Material and Its Applications. J Adv Conc Tech 1 (3): 215-230.

Lofgren I, Stang H, Olesen JF (2008) The WST method, a fracture mechanics test method for FRC. Mat Struct 41:197-211. DOI 10.1617/s11527-007-9231-3

Pereira EB, Fischer G, Barros JAO, Lepech M (2010) Crack formation and tensile stress-crack opening behavior of fiber reinforced cementitious composites (SHCC). In Oh BH, Choi OC, Chung L (eds). Proceedings of FraMCoS-7, Jeju, Korea, May 23-28.

Rossi P (1997) High performance multimodal fiber reinforced cement composites (HPMFRCC): The LCPC experience. ACI Mat J 94:478-483.

Stang H, Li VC, Krenchel H (1995) Design and structural applications of stress-crack width relations in fibre reinforced concrete. Mat Struct 28:210-219.

Zhang J, Leung CKY, Xu S (2010) Evaluation of fracture parameters of concrete from bending test using inverse analysis approach. Mat Struct 43:857-874. 